\title{
Prevalence and mortality of cryptococcal disease in adults with advanced HIV in an urban tertiary hospital in Sierra Leone: a prospective study
}

Sulaiman Lakoh ${ }^{1,2^{*}+}$ (D), Hannah Rickman ${ }^{3,4,5+}$, Momodu Sesay ${ }^{6}$, Sartie Kenneh², Rachael Burke ${ }^{3,4,5}$, Mamadu Baldeh², Darlinda F. Jiba², Yusuf S. Tejan², Sonia Boyle², Comfort Koroma², Gibrilla F. Deen ${ }^{1,2}$ and Fenella Beynon ${ }^{3,4,5}$

\begin{abstract}
Background: The global annual estimate for cryptococcal disease-related deaths exceeds 180,000, with three fourth occurring in sub-Saharan Africa. The World Health Organization (WHO) recommends cryptococcal antigen (CrAg) screening in all HIV patients with CD4 count $<100 / \mu$ l. As there is no previous published study on the burden and impact of cryptococcal disease in Sierra Leone, research is needed to inform public health policies. We aimed to establish the seroprevalence and mortality of cryptococcal disease in adults with advanced HIV attending an urban tertiary hospital in Sierra Leone.
\end{abstract}

Methods: A prospective cohort study design was used to screen consecutive adult (18 years or older) HIV patients at Connaught Hospital in Freetown, Sierra Leone with CD4 count below 100 cells $/ \mathrm{mm}^{3}$ from January to April 2018. Participants received a blood CrAg lateral flow assay (IMMY, Oklahoma, USA). All participants with a positive serum CrAg had lumbar puncture and cerebrospinal fluid (CSF) CrAg assay, and those with cryptococcal diseases had fluconazole monotherapy with 8 weeks followed up. Data were entered into Excel and analysed in Stata version 13.0. Proportions, median and interquartile ranges were used to summarise the data. Fisher's exact test was used to compare categorical variables.

Results: A total of 170 patients, with median age of 36 (IQR 30-43) and median CD4 count of $45 \mathrm{cells} / \mathrm{mm}^{3}$ (IQR 23-63) were screened. At the time of enrolment, 54\% were inpatients, 51\% were newly diagnosed with HIV, and $56 \%$ were either ART-naïve or newly initiated ( $\leq 30$ days). Eight participants had a positive blood CrAg, giving a prevalence of 4.7\% (95\% Cl: 2.4-9.2\%). Of those with a positive CrAg, CSF CrAg was positive in five (62.5\%). Five (62.5\%) CrAg-positive participants died within the first month, while the remaining three were alive and established on ART at 8 weeks.

(Continued on next page)

\footnotetext{
*Correspondence: lakoh2009@gmail.com

†'Sulaiman Lakoh and Hannah Rickman contributed equally to this manuscript as joint first authors.

'Department of Internal Medicine, College of Medicine and Allied Health Sciences, University of Sierra Leone, Freetown, Sierra Leone

${ }^{2}$ Ministry of Health and Sanitation, Government of Sierra Leone, Freetown, Sierra Leone

Full list of author information is available at the end of the article
}

(c) The Author(s). 2020 Open Access This article is distributed under the terms of the Creative Commons Attribution 4.0 International License (http://creativecommons.org/licenses/by/4.0/), which permits unrestricted use, distribution, and reproduction in any medium, provided you give appropriate credit to the original author(s) and the source, provide a link to the Creative Commons license, and indicate if changes were made. The Creative Commons Public Domain Dedication waiver (http://creativecommons.org/publicdomain/zero/1.0/) applies to the data made available in this article, unless otherwise stated. 
(Continued from previous page)

Conclusion: A substantial prevalence of cryptococcal antigenaemia and poor outcome of cryptococcal disease were demonstrated in our study. The high mortality suggests a need for the HIV programme to formulate and implement policies on screening and pre-emptive fluconazole therapy for all adults with advanced HIV in Sierra Leone, and advocate for affordable access to effective antifungal therapies.

Keywords: HIV/Cryptococcal antigenaemia/ART/newly diagnosed,

\section{Background}

Disseminated cryptococcal disease, most commonly manifesting as cryptococcal meningitis, is a serious opportunistic infection in people with advanced HIV infection. Globally it is estimated to kill over 180,000 people per year, with $75 \%$ of deaths occurring in sub-Saharan Africa [1]. Mortality of cryptococcal meningitis in lowincome countries may exceed $70 \%$ [1].

In addition to scaling up HIV testing and antiretroviral therapy (ART) coverage, 2018 WHO guidelines recommend two strategies for the prevention of cryptococcal meningitis [2]. The preferred option is screening patients with CD4 below 100 for cryptococcal antigen (CrAg), which has been shown to be effective and cost-effective in a variety of low-resource settings [3]' [4]. However, an alternative strategy of fluconazole primary prophylaxis for patients with CD4 below 100 may also be effective in reducing cryptococcal meningitis mortality $[5,6]$ and is recommended where CrAg screening is not available [2].

Sierra Leone has an estimated HIV prevalence of $1.5 \%$ [7]. Despite recent ART scale-up there continues to be a significant burden of undiagnosed infection, treatment failure, and advanced disease. There is a significant human resource for health gap [8] and the majority of HIV care is nurse-led. The only available treatment for cryptococcal meningitis is fluconazole monotherapy, which is known to be associated with inferior outcomes compared to highly recommended antifungal regimens [9].

There is no previous published study of the prevalence of cryptococcal disease and related mortality in Sierra Leone, or the prevalence of cryptococcal antigen in people living with HIV (PLHIV). We, therefore, performed a cross-sectional study to analyse a pilot programme of cryptococcal antigen screening in inpatients and outpatients PLHIVs with CD4 cell count $<100 / \mu$ l an urban referral hospital in Sierra Leone. We aimed to establish the prevalence of cryptococcal antigenaemia and diseases and to assess cryptococcal disease-related mortality in people living with HIV in this setting.

\section{Methods}

\section{Study setting and design}

This was a prospective cohort study based in Sierra Leone's tertiary adult referral hospital in Freetown. This acts as the central hub for HIV care in Sierra Leone, as well as a referral site for other patients nationwide. The HIV clinic serves approximately 4000 patients.

\section{Participant selection}

Participants were recruited sequentially over 16 weeks from January to April 2018. Participants were recruited from the CD4 laboratory based in the HIV clinic, with all patients aged 18 years or older with a CD4 less than 100 cells $/ \mathrm{mm}^{3}$ eligible for inclusion. This included inpatients and outpatients, and both newly diagnosed patients and those having CD4 cell count measured for disease monitoring or investigation of suspected opportunistic infections. Exclusion criteria were those under 18, patients with confirmed previous cryptococcal disease, and those who declined consent.

\section{Screening procedure}

All participants with positive cryptococcal antigen were reviewed by a doctor, had a lumbar puncture unless contraindicated, and followed up for survival for a minimum of 8 weeks. Participants with asymptomatic cryptococcal antigenemia were treated with oral fluconazole monotherapy @ a dose of $800 \mathrm{mg}$ daily for 2 weeks followed by $400 \mathrm{mg}$ daily for 8 weeks and then $200 \mathrm{mg}$ daily longterm (for at least 1 year, until CD4 > 200.) ART initiation was deferred to 2 weeks. Participants with cryptococcal meningitis were treated with fluconazole monotherapy according to national policy; oral/intravenous fluconazole monotherapy @ 1200 mg daily for 2 weeks, followed by an oral dose of $800 \mathrm{mg}$ daily for 8 weeks and then continued on $200 \mathrm{mg}$ daily for at least 1 year until CD4 count $>200 / \mathrm{mm}^{3}$. ART initiation was deferred at 4-6 weeks.

At the time of study initiation, this regimen was listed as an acceptable, though not a preferred regimen in the WHO guidelines, and is the only treatment available for cryptococcal meningitis in Sierra Leone.

\section{Clinical procedure}

Demographic and clinical information was collected from participants using a standardised data collection form by a trained research nurse and cross-checked with clinical records. CrAg-positive patients were followed up by medical and nursing members of the research team, but responsibility for patient management was with the 
admitting medical consultant (inpatients) and HIV clinic staff (outpatients).

Vital status was confirmed by review or discussion with the participant or a relative by telephone (where consent was given). A post-mortem was not available, and it was therefore not possible to definitively confirm the cause of death; this was based on the clinical impression of the medical team.

\section{Laboratory procedures}

CD4 was measured using a point-of-care PIMA analyser using whole blood from fingerpick or EDTA samples. Whole blood CrAg was measured using a commercially available Lateral Flow Assay (IMMY, Oklahoma, USA); quantitative measurement was not performed.

As a minimum, all patients had a CSF CrAg test, and measurement of opening pressure using sterile intravenous giving sets, or approximation using CSF drip rates (manometers not available) [10]. Other CSF analysis depended on the availability of tests; where formal laboratory testing was not available, some CSF parameters were approximated using urine dipstick testing of CSF [11].

\section{Sample size}

The study was intended to follow the duration of a 16week screening pilot. If the prevalence of cryptococcal antigen is $10 \%$ (similar to studies in Nigeria, the nearest geographical data), the sample size for 95\% confidence and $5 \%$ accuracy was 138 patients. In 16 weeks, it was estimated that 160 patients would attend with CD $4<100$.

\section{Statistical analysis}

Data were entered into Excel and analysed in Stata version 13.0(College Station, Texas, USA: StataCorp LP). Data were summarised using proportions (categorical data) and median and interquartile ranges (non-parametric continuous data). Fisher's exact test was used to compare categorical variables.

\section{Ethical issues}

This was an observational study in the context of the implementation of a WHO-recommended intervention (cryptococcal antigen screening). When patients had the capacity, informed written consent was sought for completion of a questionnaire and examination, and the use of anonymised clinical and demographic data. For all patients, the consent form was explained verbally in their local languages. Where patients were illiterate, the study and the consent form content were explained to them verbally and they indicated consent through a witnessed fingerprint. Patients who declined consent were still eligible for CrAg testing, but their data was not included in the analysis.
Some eligible participants lacked capacity as a result of suspected neurological opportunistic infections. The inclusion of this group was important in understanding the prevalence of cryptococcal infection in the study population. It was not felt ethical to seek consent from a representative as this would have involved the disclosure of HIV status; less than a third of people living with HIV in Sierra Leone have disclosed their status to adult relatives and levels of stigma are extremely high [12]. As study procedures posed minimal risk to participants, anonymised data from patients who were unable to consent were therefore also included. Consent was sought retrospectively from participants who regained capacity. All participants were free to withdraw at any time, and data were stored confidentially and anonymously.

All study protocols were approved by the Sierra Leone Ethics and Scientific Review Committee.

\section{Results}

In total, 170 participants were screened (Table 1). 59\% were female, with a median age of 36 years (IQR 30-43). At the time of testing, $54 \%$ were inpatients. $51 \%$ were newly diagnosed with HIV (defined as diagnosed with HIV less than 90 days prior to enrolment), and 56\% were ART-naïve or had been newly initiated on ART (within the past 30 days), whereas $44 \%$ were ART-experienced. The median CD4 cell count was 45 cells $/ \mathrm{mm}^{3}$ (IQR 23-63).

Eight participants were $\mathrm{CrAg}$ positive, giving a prevalence of $4.7 \%$ (95\% confidence interval 2.4-9.2). While this study was not powered to look at predictors of $\mathrm{CrAg}$ positivity, there were no significant predictors of antigenaemia, although there was a trend towards a higher prevalence amongst inpatients of $6.6 \%$ (95\% CI 2.9-14.1) vs $1.8 \%$ in outpatients (95\% CI 0.6-9.8) ( $p=0.287$ ).

Of the eight participants who were $\mathrm{CrAg}$ positive, seven (87.5\%) were female, with a median age of 35 (IQR 27.5-37) (Table 2). Six (75\%) were inpatients at the time of diagnosis, and six (75\%) were newly HIVdiagnosed and ART-naïve. The remaining two participants were taking ART at the time of CD4 testing (viral load results unavailable). On review by a clinician, seven $(87.5 \%)$ had a history of at least one of headache, confusion or decreased conscious level $(75,50$, and $50 \%$ respectively). Four (50\%) had features of meningitis on examination. All eight participants underwent a lumbar puncture. Four (50\%) had a CSF opening pressure greater than $20 \mathrm{cmCSF}$, and five $(62.5 \%)$ had a positive CSF CrAg.

The three participants with cryptococcal antigenaemia and a negative CSF CrAg were treated with oral fluconazole. CSF fungal cultures or polymerase chain reaction (PCR) were not performed as facilities for these techniques are not locally available. Two (67\%) died as hospital inpatients at 0 - and 1-days post-CrAg; both were 
Table 1 Prevalence of cryptococcal antigenaemia (CrAg) by demographic and clinical characteristics

\begin{tabular}{|c|c|c|c|c|}
\hline & $\begin{array}{l}\text { Total number tested for } \\
\text { CrAg (\% of total) }\end{array}$ & Number $\mathrm{CrAg}$ positive & Prevalence of $\mathrm{CrAg} \%(95 \% \mathrm{Cl})$ & $P$-value (Fisher's exact) \\
\hline Total & 170 & 8 & $4.7(2.4-9.2)$ & \\
\hline \multicolumn{4}{|l|}{ Sex } & 0.144 \\
\hline Male & 69 (40.6) & 1 & $1.4(0.2-10.0)$ & \\
\hline Female & $101(59.4)$ & 7 & $6.9(3.3-14.0)$ & \\
\hline \multicolumn{4}{|l|}{ Age (years) } & 1.000 \\
\hline$<35$ & $64(37.6)$ & 3 & $4.7(1.5-13.9)$ & \\
\hline$\geq 35$ & $106(62.4)$ & 5 & $4.7(1.9-11.0)$ & \\
\hline \multicolumn{4}{|l|}{ Admission status } & 0.287 \\
\hline Outpatient & $79(46.5)$ & 2 & $2.5(0.6-9.8)$ & \\
\hline Inpatient & $91(53.5)$ & 6 & $6.6(2.9-14.1)$ & \\
\hline \multicolumn{4}{|l|}{ CD4 (cells $\left./ \mathrm{mm}^{3}\right)$} & 1.000 \\
\hline$<50$ & $96(56.5)$ & 5 & $5.2(0.7-9.7)$ & \\
\hline$\geq 50$ & $74(43.5)$ & 3 & $4.1(0.0-8.7)$ & \\
\hline \multicolumn{4}{|l|}{ Time since HIV diagnosis (days) } & 0.278 \\
\hline < 90 "newly diagnosed" & $86(50.6)$ & 6 & $7.0(3.1-14.9)$ & \\
\hline$\geq 90$ & $84(49.4)$ & 2 & $2.4(0.6-9.5)$ & \\
\hline \multicolumn{4}{|c|}{ Previous antiretroviral therapy $(\mathrm{ART})^{\mathrm{a}}$} & 0.468 \\
\hline ART naïve or newly-initiated & $91(56.2)$ & 6 & $6.6(2.9-14.1)$ & \\
\hline ART experienced & $71(43.8)$ & 2 & $2.8(0.7-10.9)$ & \\
\hline \multicolumn{4}{|l|}{ On ART at time of recruitment ${ }^{a}$} & 1.000 \\
\hline Yes & $43(26.9)$ & 2 & $4.7(1.1-17.6)$ & \\
\hline No & $117(73.1)$ & 6 & $5.1(2.3-11.1)$ & \\
\hline
\end{tabular}

${ }^{a}$ It was not possible to determine ART history for 8 participants (4.7\%), or current ART status for 10 participants (5.9\%); all of these were CrAg negative

confirmed as TB coinfected and neither participant was the cause of death felt clinically to be cryptococcal disease. The remaining participant was alive and established on ART at 8 weeks of follow-up. All five participants with cryptococcal meningitis were treated with high-dose fluconazole, and all received at least two therapeutic lumbar punctures. Three (60\%) died at 3, 5 and 20 days after $\mathrm{CrAg}$ testing. Two (40\%) were alive and established on ART at 8 weeks of follow-up.

\section{Discussion}

Our study has demonstrated a prevalence of 4.7\% (95\% confidence interval 2.4-9.2) of cryptococcal infection among people with advanced HIV in a large tertiary hospital in Sierra Leone. To our knowledge, this is the first assessment of cryptococcal antigenaemia and cryptococcal disease-related mortality in this setting. This prevalence exceeds the previously recommended WHO threshold for the institution of routine screening for cryptococcal diseases [13]. In other published studies from West Africa, CrAg seroprevalence in PLHIV with $\mathrm{CD} 4<100$ cells $/ \mathrm{mm}^{3}$ ranges from 2 to $21 \%$ [14-18]. However, heterogeneity between study populations (for example, whether they include only newly-diagnosed ART-naïve individuals, and/or only outpatients) and evidence of regional variation [17] prohibits direct comparison.

It was feasible to conduct $\mathrm{CrAg}$ screening in this setting. Currently, there is no national policy on diagnosis, management and prevention of cryptococcal disease in Sierra Leone, but 2018 WHO guideline on cryptococcal diseases strongly recommends $\mathrm{CrAg}$ screening for adults and adolescents with CD4 cell count $<100$ cells $/ \mathrm{mm}^{3}$ [2]. This intervention has been shown to be costeffective and effective in reducing cryptococcal-related mortality $[4,19]$, and it is hoped that this evidence will contribute to wider adoption in Sierra Leone.

The overall mortality among $\mathrm{CrAg}$ positive participants within 8 weeks of follow up was $62.5 \%$. Sadly, this is comparable to other studies from the region [14] and to the overall estimate of $70 \%$ one-year mortality for people in LMICs with cryptococcal meningitis [1]. One contributor to the high mortality in our study is likely to be the use of fluconazole monotherapy in the treatment of cryptococcal meningitis. At the time of study initiation, this was still listed as an alternative treatment by 


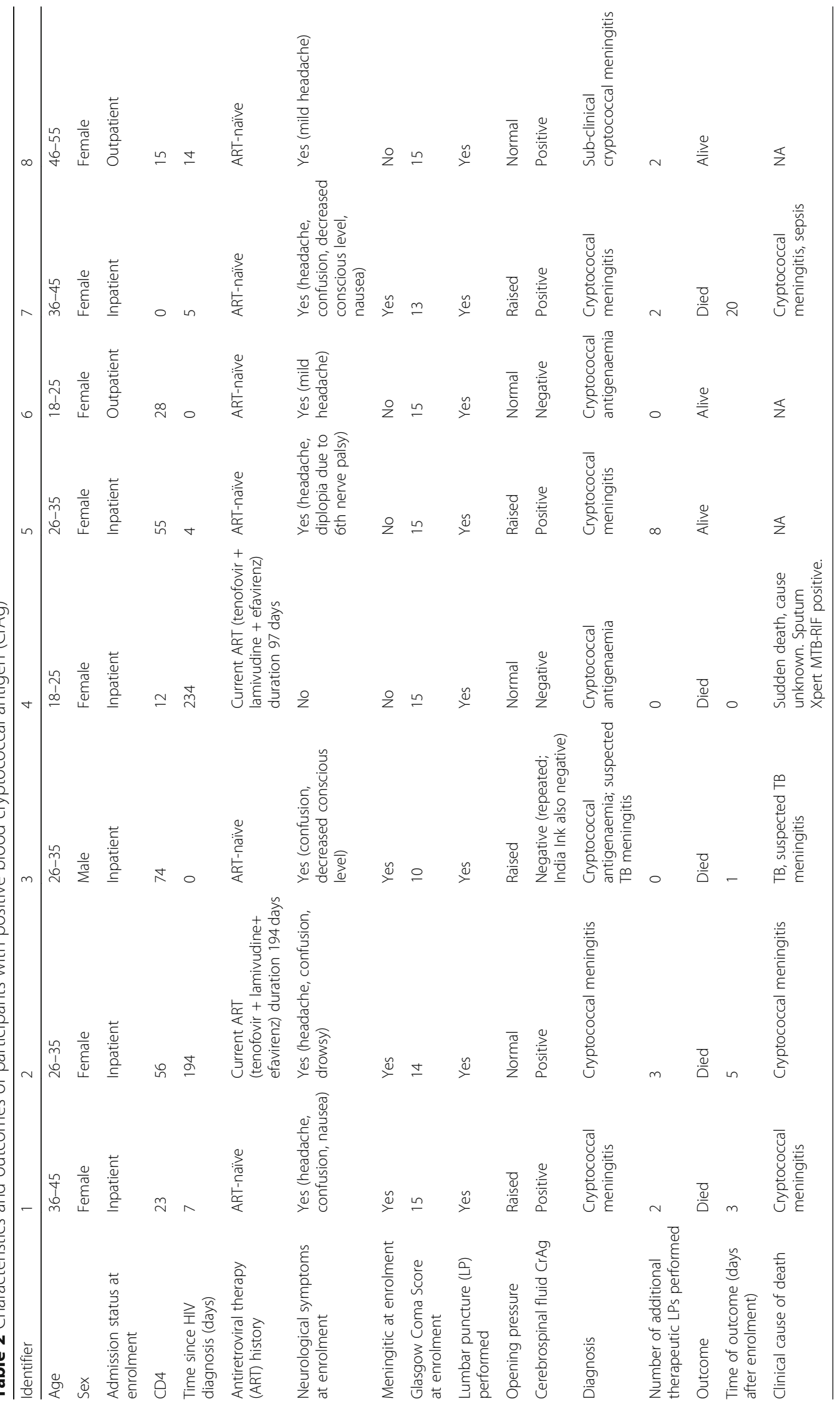


the WHO guidelines but is no longer recommended for the treatment of cryptococcal meningitis given the abundance of evidence for superior antifungal regimens $[2,20]$. Despite the new WHO recommendation of dual antifungal therapy as an induction regimen for cryptoccocal meningitis [2], fluconazole remains the only available treatment for cryptococcal disease in Sierra Leone. We, therefore, add our voices to those advocating for effective treatments for cryptococcal disease to be made more accessible to PLHIV in low-income countries [21]. Routinely screening patients with advanced HIV disease for CrAg as part of their pre-ART interventions and the provision of appropriate dual antifungal regimen should be instituted in Sierra Leone and similar countries to prevent the unwarranted morbidity and mortality [2, 22].

Given that several of the deceased participants were felt clinically to have a cause of death other than cryptococcal disease, it is important to deliver a "package" of care to those with advanced HIV (CD4 cell count $<200$ cells $/ \mathrm{mm}^{3}$ ) [23] which includes $\mathrm{CrAg}$ screening and other aspects including tuberculosis screening and prevention, and rapid ART initiation. Sierra Leone has recently operationalised a differentiated HIV service delivery guide which includes provisions directed towards people with advanced HIV.

Additional measures are also needed to reduce cryptococcal mortality in Sierra Leone. In a recent study, $21 \%$ of people with newly-diagnosed HIV in Freetown had a CD4 cell count below 100 cells $/ \mathrm{mm}^{3}$, suggesting a need for earlier HIV testing, particularly given the limited resources for managing opportunistic infections [24]. Similar to other studies in sub-Saharan Africa [25], a significant proportion of participants in this study were ARTexperienced, either with presumed treatment failure, poor adherence, or loss from care. This calls for prudent programmatic activities to improve treatment adherence. Many studies of cryptococcosis prevention exclusively focus on ART-naivve populations, but our finding that two (25\%) of those with cryptococcal antigenaemia were ARTexperienced suggests that $\mathrm{CrAg}$ screening should not be restricted to newly diagnosed HIV. PLHIV with virologic or immunologic treatment failure and those with symptoms who report after treatment interruptions should be considered for routine $\mathrm{CrAg}$ screening if their $\mathrm{CD} 4$ cell count is $<200$ cells $/ \mathrm{mm}^{3}$.

Our study has several limitations. First, as a single-site study in a tertiary hospital, it may not be representative of Sierra Leone with respect to both patient characteristics and feasibility of implementing screening. Secondly, $54 \%$ of participants were inpatients and those who presented with neurological symptoms were not excluded. Therefore, they may not truly represent the impact of a screening programme (ideally targeting asymptomatic outpatients.) However, this study was conducted in the context of implementation of a reflexive $\mathrm{CrAg}$ testing programme, and the study population does reflect those tested for CD4 cell count at the centre. Lack of fungal cultures and other diagnostic tests did not make it possible to confirm diagnoses of cryptococcosis or meningitis; nor was it possible to definitively establish the cause of death in deceased $\mathrm{CrAg}$-positive participants.

\section{Conclusion}

Our study demonstrated a significant burden of cryptococcal disease at Connaught Hospital in Sierra Leone. We recommend for the national HIV programme and its partners to formulate and implement policies on screening and pre-emptive fluconazole therapy for cryptococcal diseases as part of a package of differentiated care for people with advanced HIV, in conjunction with programmatic strategies to improve HIV diagnosis and virological suppression. Affordable access to adequate antifungal regimens is required to improve outcomes for cryptococcal disease in sub-Saharan Africa.

Finally, antiretroviral treatment interruptions among the cohort of patients in this study are high. In order to prevent this, appropriate psychosocial counselling techniques and other programmatic strategies are needed.

\section{Abbreviations \\ ART: Antiretroviral therapy; ARVs: Antiretrovirals; CD4: Cluster determinant 4; CrAg: Cryptococcal Antigen; CSF: Cerebrospinal fluid; HIV: Human Immunodeficiency Virus; LMIC: Low- and Middle-Income Countries; PLHIV: People Living with HIV; SLESRC: Sierra Leone Ethics and Scientific Review Committee; WHO: World Health Organization}

\section{Acknowledgements}

We thank the management and all the medical teams of Connaught Hospital. We acknowledge the staff of the medical wards and HIV Clinic especially Miss Isata Kamara. We are grateful to the patients and their caregivers.

\section{Authors' contributions}

S.L., H.R., M.S., F.B., R.B., S.K., and G.F.D., designed the research; H.R., D.F.J. M.B. S.B., C.K., Y.S.T., F.B., and S.L. collected and analysed the data; and S.L., H.R. F.B., G.F.D., S.K., D.F.J, M.S., S.B., M.B., C.K., and Y.S.T. wrote the paper. All authors read and approved the final manuscript.

\section{Author's information}

The corresponding author, S. L, is an Infectious Disease Senior Resident and a Lecturer in the Infectious Disease Division of the Department of Medicine, University of Sierra Leone. His research interests are the science and epidemiology of HIV and antimicrobial resistance.

\section{Funding}

This work was supported by King's Sierra Leone Partnership through a European and Developing Countries Clinical Trials Partnership grant for research capacity building (EDCTP-CSA-Ebola-360). Routine clinical care was provided by the Ministry of Health and Sanitation and the National AIDS Control Programme, supported by the Global Fund to fight AIDS,

Tuberculosis and Malaria.

\section{Availability of data and materials}

The data supporting this article is available in the repository of University of Sierra Leone and will be made easily available on request to the corresponding author when required. 


\section{Ethics approval and consent to participate}

Ethical approval was obtained from the Sierra Leone Ethics and Scientific Review Committee (SLESRC) of the Ministry of Health and Sanitation, Government of Sierra Leone. When patients had the capacity, informed written consent was approved by SLESRC was sought and obtained for completion of a questionnaire and examination. For all patients, the consent form was explained verbally in their local languages. Where patients were illiterate, the study and the consent form content were explained to them verbally and they indicated consent through a witnessed fingerprint as approved by SLESRC. Patients who declined consent were still eligible for CrAg testing, but their data was not included in the analysis.

Some eligible participants lacked capacity as a result of suspected neurological opportunistic infections. The inclusion of this group was important in understanding the prevalence of cryptococcal infection in the study population. It was not felt ethical to seek consent from a representative as this would have involved the disclosure of HIV status; less than a third of people living with HIV in Sierra Leone have disclosed their status to adult relatives and levels of stigma are extremely high [12]. As study procedures posed minimal risk to participants, anonymised data from patients who were unable to consent were therefore also included. Consent was sought retrospectively from participants who regained capacity. All participants were free to withdraw at any time, and data were stored confidentially and anonymously.

All study protocols (including waiver of consent from the next-of-kin and the procedure of consent from the patients that lacked the capacity) were approved by the SLERSC.

\section{Consent for publication}

Not applicable

\section{Competing interests}

The authors declare that they have no competing interest.

\section{Author details \\ 'Department of Internal Medicine, College of Medicine and Allied Health Sciences, University of Sierra Leone, Freetown, Sierra Leone. ${ }^{2}$ Ministry of Health and Sanitation, Government of Sierra Leone, Freetown, Sierra Leone. ${ }^{3}$ King's Sierra Leone Partnership, Freetown, Sierra Leone. ${ }^{4}$ King's Centre for Global Health and Health Partnerships, London, UK. ${ }^{5}$ King's College London, London, UK. ${ }^{6}$ National HIV/AIDS Secretariat, Government of Sierra Leone, Freetown, Sierra Leone.}

Received: 3 June 2019 Accepted: 6 February 2020

Published online: 14 February 2020

\section{References}

1. Rajasingham R, Smith RM, Park BJ, Jarvis JN, Govender NP, Chiller TM, et al. Global burden of disease of HIV-associated cryptococcal meningitis: an updated analysis. Lancet Infect Dis. 2017;17(8):873-81.

2. WHO | Guidelines for the diagnosis, prevention and management of cryptococcal disease in HIV-infected adults, adolescents and children. WHO. 2018; Available from: apps.who.int/iris/bitstream/handle/10665/260399/ 9789241550277-eng.pdf;jsessionid=30132897E0F565C43DF9DDFD1B0AFA3 F? sequence=1. Accessed 13 Dec 2019.

3. Meya DB, Manabe YC, Castelnuovo B, Cook BA, Ali M, Kambugu A, et al. Serum Cryptococcal antigen (CRAG) screening is a cost- effective method to prevent death in HIV- infected persons with CD4 <100/uL starting HIV therapy in resource-limited settings. Clin Infect Dis. 2010;51(4):448-55.

4. Jarvis JN, Harrison TS, Lawn SD, Meintjes G, Wood R, Cleary S. Cost effectiveness of Cryptococcal antigen screening as a strategy to prevent HIVassociated Cryptococcal meningitis in South Africa. PLoS One. 2013;8(7):1-10.

5. Hakim J, Musiime V, Szubert AJ, Mallewa J, Siika A, Agutu C, et al. Enhanced prophylaxis plus antiretroviral therapy for advanced HIV infection in Africa. N Engl J Med. 2017 Jul;377(3):233-45.

6. Parkes-Ratanshi R, Wakeham K, Levin J, Namusoke D, Whitworth J, Coutinho A, et al. Primary prophylaxis of cryptococcal disease with fluconazole in HIVpositive Ugandan adults: a double-blind, randomised, placebo-controlled trial. Lancet Infect Dis. 2011;11(12):933-41.

7. Sierra Leone Demographic and Health Survey. 2013; Available from: www. statistics.s//images/StatisticsSL/Documents/demographic_and_health_ survey_2013_final-report.pdf. Accessed 10 Feb 2020.
8. WHO | WHO Global Health Workforce Statistics. WHO. 2018; Available from: www.who.int/hrh/statistics/en/. Accessed 12 Dec 2019.

9. Gaskell KM, Rothe C, Gnanadurai R, Goodson P, Jassi C, Heyderman RS, et al. A Prospective Study of Mortality from Cryptococcal Meningitis following Treatment Induction with $1200 \mathrm{mg}$ Oral Fluconazole in Blantyre, Malawi. Filler SG, editor. PLoS One. 2014;9(11):e110285.

10. Boyles TH, Gatley E, Wasserman S, Meintjes G. Brief report. JAIDS J Acquir Immune Defic Syndr. 2017;74(3):e64-6.

11. Molyneux E. Where there is no laboratory, a urine patch test helps diagnose meningitis. J Neurosci Rural Pract. 2013 Apr;4(2):117-8.

12. World Health Organization. Sierra Leone Sierra Leone. Time. 2016, 2003;(January):-4.

13. Organization WH. Rapid advice: diagnosis, prevention and management of cryptococcal disease in HIV-infected adults, adolescents and children: December 2011. Geneva World Heal Organ. 2011;(December) Available from: http://apps.who.int/iris/handle/10665/44786.

14. Thomsen D, Hviid CJ, Hønge BL, Medina C, da Silva Té D, Correira FG, et al. Increased mortality among HIV infected patients with cryptococcal antigenemia in Guinea-Bissau. Pan Afr Med J. 2018.

15. Osazuwa F, Dirisu JO, Okuonghae PE, Ugbebor O. Screening for cryptococcal antigenemia in anti-retroviral naïve AIDS patients in Benin City, Nigeria. Oman Med J. 2012;27(3):228-31.

16. Oladele RO, Akanmu AS, Nwosu AO, Ogunsola FT, Richardson MD, Denning DW. Cryptococcal antigenemia in Nigerian patients with advanced human immunodeficiency virus: Influence of antiretroviral therapy adherence. Open Forum Infect Dis. 2016;3(2):ofw055.

17. Ezeanolue EE, Nwizu C, Greene GS, Amusu O, Chukwuka C, Ndembi N, et al. Brief report: geographical variation in prevalence of Cryptococcal Antigenemia among HIV-infected, treatment-naive patients in Nigeria: a multicenter crosssectional study. J Acquir Immune Defic Syndr. 2016;73(1):117-21.

18. Mamoojee Y, Shakoor S, Gorton RL, Sarfo S, Appiah LT, Norman B, et al. Short communication: low seroprevalence of cryptococcal antigenaemia in patients with advanced HIV infection enrolling in an antiretroviral programme in Ghana. Tropical Med Int Health. 2011;16(1):53-6.

19. Meya D, Rajasingham R, Nalintya E, Tenforde M, Jarvis JN. Preventing Cryptococcosis - shifting the paradigm in the era of highly active antiretroviral therapy. Curr Trop Med Reports. 2015;2(2):81-9.

20. WHO. Rapid Advice: Diagnosis, Prevention and Management of Cryptococcal Disease in HIV-Infected Adults, Adolescents and Children. 2011. Available from: apps.who.int/iris/bitstream/handle/10665/44786/ 9789241502979_eng.pdf?sequence=1\&isAllowed=y. Accessed 09 Feb 2020 .

21. Loyse A, Burry J, Cohn J, Ford N, Chiller T, Ribeiro I, et al. Leave no one behind: response to new evidence and guidelines for the management of cryptococcal meningitis in low-income and middle-income countries. Lancet Infect Dis. 2018; Available from: www.dndi.org/wp-content/ uploads/2018/10/Loyse_Response_Evidence_Guidelines_ CryptococcalMeningitis_LancetID_2018.pdf.

22. Vaidhya SA. Combination versus Monotherapy for the treatment of HIV associated Cryptococcal meningitis. J Clin Diagnostic Res [Internet]. 2015;50(2): 16-8 Available from: http://jcdr.net/article_fulltext.asp?issn=0973-709x\&year= 2015\&volume $=9 \&$ issue $=2 \&$ page $=0 C 14 \& i s s n=0973-709 \times \& i d=5601$.

23. WHO. HIV Treatment. Guidelines for managing advanced HIV disease and rapid initiation of ARV; 2017. p. 2-5. Available from: http://apps.who.int/iris/ bitstream/10665/255885/1/WHO-HIV-2017.18-eng.pdf?ua=1

24. Yendewa GA, Poveda E, Lakoh S, Yendewa SA, Jiba DF, Salgado-Barreira A, et al. High prevalence of late-stage disease in newly diagnosed human immunodeficiency virus patients in Sierra Leone. Open Forum Infect Dis. 2018;5(9):7-10 Available from: https://academic.oup.com/ofid/article/doi/1 0.1093/ofid/ofy208/5078457.

25. Ousley J, Niyibizi AA, Wanjala S, Vandenbulcke A, Kirubi B, Omwoyo W, et al. High proportions of patients with advanced HIV are antiretroviral therapy experienced: hospitalization outcomes from 2 sub-Saharan African sites. Clin Infect Dis. 2018;66(August):S126-32.

\section{Publisher's Note}

Springer Nature remains neutral with regard to jurisdictional claims in published maps and institutional affiliations. 